\title{
Peripheral blood picture and aminotransferase activity in children with newly diagnosed Graves' disease at baseline and after the initiation of antithyroid drug therapy
}

\author{
DOROTA ARTEMNIAK-WOJTOWICZ, EWELINA WITKOWSKA-SEDEK, ADA BOROWIEC, \\ BEATA PYRŻAK \\ Department of Paediatrics and Endocrinology, Medical University of Warsaw, Warsaw, Poland
}

\begin{abstract}
Aim of the study: Assessment of the peripheral blood picture and aminotransferase activity in children with newly diagnosed Graves' disease (GD) at baseline and 4-6 weeks after the initiation of antithyroid drug (ATD) therapy.

Material and methods: Data of 59 children were assessed retrospectively. Baseline analysis included concentrations of thyroid-stimulating hormone (TSH), free triiodothyronine (fT3), free thyroxine (fT4), TSH receptor antibodies (TSH-R Ab), complete blood cell count (CBC), aspartate (AST) and alanine aminotransferase (ALT) activity. Reassessment of $C B C$ and aminotransferase activity was performed 4-6 weeks after the initiation of ATD therapy.

Results: Significant decreases in the neutrophil count, $M C V$, haemoglobin $(\mathrm{Hgb})$, red blood cell $(R B C)$ count, white blood cell (WBC) count and platelet (PLT) count were found in $37.3 \%, 32.2 \%$, $22 \%, 13.6 \%, 8.5 \%$ and $5 \%$ of untreated patients, respectively. Increased baseline ALT and AST activity was observed in $44 \%$ and $32.2 \%$ of children, respectively. Initiation of ATD therapy led to significant changes in $\mathrm{Hgb}, \mathrm{RBC}$ and PLT count, RDW and ALT activity. Negative associations between TSH-R $A b, T S H$ and MCV were found. ALT and AST activity were negatively related to baseline TSH levels. ALT activity was also associated with baseline fT4 and fT3.

Conclusions: The incidence of haematopoiesis and liver abnormalities in GD children seems to be similar to that reported in adult patients. The most common alterations are changes in neutrophil count, RBC parameters and ALT activity. The initiation of ATD therapy usually leads to significant improvement in those parameters.
\end{abstract}

Key words: hyperthyroidism, autoimmunity, peripheral blood cells, liver function, children.

(Centr Eur J Immunol 2019; 44 (2): 132-137)

\section{Introduction}

Graves' disease (GD) is the most common cause of hyperthyroidism in children and adolescents. It is a systemic autoimmune disease characterized by the infiltration of thyroid antigen-specific T cells into thyroid-stimulating hormone receptor (TSH-R) expressing tissues [1, 2]. Pathogenesis of GD is complex and includes both genetic and environmental factors, such as cigarette smoking, high dietary iodine intake, stress and pregnancy [2-6]. In children it may occur at any age with a peak during adolescence, and similarly to adults, it is much more frequent in girls than in boys [1]. Manifestations of GD result from stimulatory autoantibodies $(\mathrm{Ab})$ which activate TSH-R leading to uncontrolled production and secretion of thyroid hormones (TH) and to the development of the goiter [2]. Anti-thyroid peroxidase antibody and anti-thyroglobulin antibody could also be detected in patients with hyperthyroidism, but TSH-R Ab are specific biomarkers for GD $[7,8]$.

Untreated thyrotoxicosis as well as autoimmune process itself, exerts wide-ranging effects on a variety of organ systems, including the influence on haematopoiesis and liver function [9-12]. After the initiation of antithyroid drug (ATD) therapy those abnormalities are unusually reversible, but in some patients they could persist or appear owing to the negative impact of such therapy [13-18].

Correspondence: Ewelina Witkowska-Sędek, PhD, Department of Paediatrics and Endocrinology, Medical University of Warsaw,

63A Żwirki i Wigury St., 02-091 Warsaw, Poland, e-mail: ewelina.witkowska-sedek@wum.edu.pl

Submitted: 8.01.2019; Accepted: 17.04.2019 
Hyperthyroidism could result in changes in all blood cell lineages [11, 19-23]. Thyroid hormone excess due to increased tissue oxygen demands enhances erythropoiesis through hyperproliferation of immature erythroid progenitors and increased secretion of erythropoietin (EPO) by inducing erythropoietin gene expression [11]. Simultaneously, an increase in plasma volume, shorter erythrocyte life span, increases in iron turnover and iron, vitamin $B_{12}$ or folate deficiency could result in anaemia [21, 23]. On the other hand, hyperthyroidism leads to a (usually mild) decrease in total white blood cell count, neutropenia and thrombocytopenia [11, 23, 24]. Pancytopenia and autoimmune haemolytic anaemia are rare complications of thyroid hormone excess [21, 22].

The relationships between the thyroid gland and the liver are complex. Thyroid hormone excess may lead to hepatic or cholestatic liver injury, and on the other hand, liver disturbances could influence thyroid hormone metabolism and action [10, 25]. Thyroid autoimmunity such as GD can be associated with primary biliary cirrhosis or hepatitis due to autoimmune process activity [26]. The incidence of liver biochemical abnormalities in patients with untreated thyrotoxicosis varies from $15 \%$ to $79 \%$ [27]. Acceleration of cellular metabolism resulting from thyrotoxicosis may lead directly to relative hepatic anoxia, hepatocyte damage and apoptosis with subsequent serum hepatic enzymes elevation and increased susceptibility to oxidative stress. Hepatic injury manifests predominantly in serum aspartate aminotransferase (AST) and alanine aminotransferase (ALT) elevations, while cholestatic liver injury results mainly in an increase in serum alkaline phosphatase (ALP) and rarely in elevations in gamma-glutamyl transpeptidase (GGT) and bilirubin [10, 27]. In the majority of patients liver dysfunctions are self-limiting and reversible after the initiation of ATD therapy [10, 28, 29]. Rarely, the development of hepatocellular necrosis and perivascular fibrosis leads to persistent hepatitis and progressive liver failure [25, 30].

The aim of this study was to assess peripheral blood picture and aminotransferase activity in patients with newly diagnosed GD before the initiation of ATD therapy and after the first 4-6 weeks of ATD treatment.

\section{Material and methods}

The consent of the Bioethics Committee at the Medical University of Warsaw was obtained before conducting the study. We analysed retrospectively the medical data of 59 children and adolescents (median age: 15 years) hospitalized due to newly diagnosed GD in the Department of Paediatrics and Endocrinology, Medical University of Warsaw, from January 2011 to June 2018. The inclusion criterion was positive TSH-R Ab assay. Other causes of abnormalities of blood cell parameters and aminotransferase activity were excluded. Baseline analysis included lev- els of thyroid-stimulating hormone (TSH), free triiodothyronine (fT3), free thyroxine (fT4), TSH-R Ab, red blood cell (RBC) count, haemoglobin (Hgb) level, the values of mean corpuscular volume (MCV) and RBC distribution width (RDW), total white blood cell (WBC) count, neutrophil count, platelet (PLT) count and AST and ALT activity. All patients enrolled into the study were treated with standard doses of methimazole (0.5-1.0 mg/kg/day). Four-six weeks after the initiation of ATD therapy we assessed levels of TSH, fT3 and fT4, RBC count, Hgb level, $\mathrm{MCV}, \mathrm{RDW}, \mathrm{WBC}$ count, neutrophil count, PLT count and aminotransferase activity. The values of the peripheral blood cell parameters and aminotransferase activity were compared to reference ranges for age.

\section{Biochemical analyses}

Complete blood count (CBC) was measured in blood collected in EDTA samples using Sysmex XN 1000i haematological analyzer. ALT and AST activity was measured by dry chemistry method using Vitros 5600 (Ortho Clinical Diagnostics) analyzer. The serum concentrations of TSH, fT 3 and fT4 were measured by immunofluorescence method using the Architect i1000SR analyzer (Abbott Diagnostics). The TSH-R Ab levels were measured by electrochemiluminescence immunoassay (ECLIA) with the Cobas e801 analyzer.

\section{Statistical analysis}

Statistical analysis was conducted using Statistica 13.1 software. Data distribution was checked using the ShapiroWilk normality test. Comparisons between baseline and ATD treatment values of TSH, fT3, fT4, RBC count, Hgb level, MCV, RDW, WBC count, neutrophil count, PLT count, ALT and AST activity were conducted using the Wilcoxon signed-rank test for non-normally distributed data and using the T-test for normally distributed data. Correlation analysis was performed using the Pearson correlation coefficient and the Spearman correlation analysis, as appropriate. A $p$ value $<0.05$ was considered statistically significant.

\section{Results}

The study group consisted of 51 girls $(86.4 \%)$ and 8 boys (13.6\%) with newly diagnosed GD. The characteristics of all the parameters which were analysed at baseline, at diagnosis of GD, and 4-6 weeks after the initiation of ATD therapy are presented in Table 1 .

The evaluation of baseline haematological parameters revealed that the most common abnormalities in peripheral blood picture in the studied children were decreased neutrophil count found in $37.3 \%$ of patients, decreased MCV found in $32.2 \%$ and decreased Hgb levels in $22 \%$. Decreased RBC count was found in $13.6 \%$ of the studied 
Table 1. The characteristics of analysed parameters at baseline and 4-6 weeks after initiation of antithyroid drug (ATD) therapy

\begin{tabular}{lccc}
\hline Parameters & Baseline & 4-6 weeks of ATD therapy & $p$-value \\
\hline Age $(\mathrm{years})$ & $15(11.6-16.3)$ & - & - \\
\hline Height $(\mathrm{cm})$ & $162.5(154.3-169.0)$ & - & - \\
\hline Weight $(\mathrm{kg})$ & $52.9(40.7-64.0)$ & - & $<0.0001$ \\
\hline TSH $(\mu \mathrm{IU} / \mathrm{ml})$ & $0.00(0.00-0.00)$ & $0.00(0.00-0.09)$ & $<0.0001$ \\
\hline fT3 $(\mathrm{pg} / \mathrm{ml})$ & $10.98(5.91-16.39)$ & $2.77(2.31-3.29)$ & $<0.0001$ \\
\hline fT4 $(\mathrm{ng} / \mathrm{dl})$ & $2.43 \pm 0.74$ & $0.83(0.66-1.01)$ & - \\
\hline TSH-R Ab $(\mathrm{IU} / \mathrm{l})$ & $9.77(3.01-14.53)$ & - & $<0.01$ \\
\hline ALT $(\mathrm{U} / \mathrm{l})$ & $33.5(26.0-42.0$ & $31.0(24.0-36.0)$ & $\mathrm{NS}$ \\
\hline AST $(\mathrm{U} / \mathrm{l})$ & $4.78 \pm 0.46$ & $28.5(24.0-34.0)$ & $<0.01$ \\
\hline RBC $\left(\times 10^{6} / \mu \mathrm{l}\right)$ & $28.0(23.0-37.0)$ & $4.87 \pm 0.42$ & $<0.01$ \\
\hline Hgb $(\mathrm{g} / \mathrm{dl})$ & $12.76 \pm 1.19$ & $13.04 \pm 1.11$ & $\mathrm{NS}$ \\
\hline MCV & $80.2(76.0-84.0)$ & $80.15(75.9-84.5)$ & $<0.0001$ \\
\hline RDW & $13.1(12.5-13.8)$ & $14.1(13.4-14.9)$ & $\mathrm{NS}$ \\
\hline WBC $\left(\times 10^{3} / \mu \mathrm{l}\right)$ & $6.7(5.3-7.9)$ & $6.9(5.9-7.7)$ & $\mathrm{NS}$ \\
\hline Neutrophils $\left(\times 10^{3} / \mu \mathrm{l}\right)$ & $2.9(2.0-4.5)$ & $3.6(2.9-4.5)$ & $<0.0001$ \\
\hline PLT $\left(\times 10^{3} / \mu \mathrm{l}\right)$ & $257.0(218.0-287.0)$ & $277.5(248.5-319.5)$ & \\
\hline
\end{tabular}

Data are presented as mean \pm standard deviation $(S D)$ or median with the interquartile range, as appropriate, TSH - thyroid-stimulating hormone, fT3 - free triiodothyronine, fT4 - free thyroxine, TSH-R Ab-TSH receptor antibodies, ALT - alanine aminotransferase activity, AST - aspartate aminotransferase activity, $R B C$ - red blood cell count, $\mathrm{Hg}$ - haemoglobin concentration, $M C V$ - mean corpuscular volume, $R D W-R B C$ distribution width, WBC - white blood cell count, PLT - platelet count, NS - not significant

group, decreased WBC count in $8.5 \%$ and decreased PLT count in $5 \%$. In contrast to the above-mentioned parameters, baseline RDW had a tendency to be higher than normal values and in $15.3 \%$ of patients exceeded the upper normal range. In most cases all those abnormalities were mild and none of the patients had severe neutropenia, anaemia or thrombocytopenia. Peripheral blood picture reassessment performed 4-6 weeks after the initiation of ATD therapy showed statistically significant changes in most of the analysed parameters compared to baseline values - decreased $\mathrm{Hgb}$ concentration was found in $10 \%$ of patients $(p<0.01)$, decreased RBC count in $3.6 \%(p<0.01)$, decreased PLT count in $1.8 \%(p<0.0001)$ and increased RDW in $51.3 \%$ of patients $(p<0.0001)$. The values of $\mathrm{MCV}, \mathrm{WBC}$ and neutrophil count did not change significantly and remained decreased in $35.7 \%, 8.9 \%$ and $10.7 \%$ of patients, respectively.

Simultaneously, increased baseline ALT activity was found in $44 \%$ of patients, but in most cases it was slightly above the upper limit of the normal range. In $6.8 \%$ of patients, baseline ALT activity exceeded the upper limit of the range more than twice. Baseline AST activity was increased in $32.2 \%$ of the studied group, but its value was 2 times higher than the upper limit of the reference value only in 5\% of patients. Four-six weeks after the initiation of ATD therapy, increased activity of both ALT and AST was observed in $22 \%$ of patients, but it did not exceed the upper limit of the range more than twice in any of the patients. Statistical analysis revealed that only the decrease in ALT activity was statistically significant $(p<0.01)$. We also noticed that in some patients, both at baseline and during ATD therapy, haematological and liver function abnormalities coexisted, but we did not investigate any potential reasons for that.

Our further analysis focused on the assessment of the relationships between baseline levels of TSH, free $\mathrm{TH}$ and TSH-R Ab and baseline and treatment values of all the above-mentioned haematological parameters and aminotransferase activity.

Correlation analysis revealed statistically significant negative relationships between TSH-R Ab levels and MCV, both at baseline $(r=-0.40, p<0.01)$ and during ATD therapy $(r=-0.45, p<0.01)$. There were no associations between baseline TSH-R Ab and any other haematological parameters. Similar analysis including baseline TSH and free TH concentrations and peripheral blood parameters showed only a significant association between baseline TSH level and baseline MCV $(r=0.36, p<0.01)$. Baseline free $\mathrm{TH}$ levels were not associated with any of the haematological parameters.

Our further analysis, which included aminotransferases activity, showed that TSH levels were negatively related 
to ALT activity, both at baseline $(r=-0.33, p<0.05)$ and during ATD therapy $(r=-0.29, p<0.05)$. The association between TSH levels and AST activity was almost significant at baseline $(p=0.054)$ and statistically significant during ATD therapy $(r=-0.32, p<0.05)$. We also found significant positive relationships between baseline free $\mathrm{TH}$ and baseline ALT activity $(r=0.32, p<0.05$ for fT 4 and $p=0.0503$ for fT3). We did not find any relationships between baseline TSH-R Ab and ALT or AST activity.

\section{Discussion}

Changes in haematopoiesis and liver metabolism are the most common dysfunctions reported in untreated thyrotoxicosis [9-12, 23, 25, 27]. The incidence of haematopoiesis disorders in patients with GD varied between studies, but anaemia seemed to be the most common, and its incidence is estimated at $10-34 \%$ of patients, while the incidence of leukopenia and thrombocytopenia is estimated to be less than $10 \%$ [31, 32]. Pancytopenia and autoimmune haemolytic anaemia are very rare complications of GD [21, 22, 32]. In our study, we evaluated the incidence of haematopoiesis disorders in children and adolescents with newly diagnosed GD and we analysed early changes in the peripheral blood picture observed in those patients 4-6 weeks after the initiation of ATD therapy.

In our study group, the most common haematologic abnormalities observed at diagnosis of GD, were decreases in neutrophil count, MCV and Hgb levels, which were found in $37.3 \%, 32.2 \%$ and $22 \%$ of , respectively. Reduced RBC count was observed in $13.6 \%$ of children, decreased WBC count in less than $10 \%$ and decreased PLT count was found only in 5\% of patients. Increased RDW was found in $15.3 \%$ of patients before ATD therapy. We did not find any significant correlations between baseline autoimmune process activity characterized by TSH-R Ab levels and any of the evaluated haematologic parameters except MCV both before and during ATD therapy. After the first 4-6 weeks of ATD therapy, Hgb levels, RBC count, RDW values and PLT count increased significantly, while MCV, WBC and neutrophil count did not change significantly compared to baseline values.

In the literature, data concerning haematologic abnormalities in children and adolescents with GD, in contrast to a number of studies including adult population, are scarce. The study by Gianoukakis et al. [33] showed that $33 \%$ of adult patients with newly diagnosed GD presented anaemia, but the incidence of anaemia not attributable to other causes (GD anaemia) was $22 \%$. The authors also noticed that anaemia was more than twice as frequent in men as in women and was associated with higher, but within normal reference limits, mean EPO levels found in those patients in contrast to patients without anaemia. Hgb concentrations correlated inversely with EPO levels and that relationship persisted also after multivariate adjustment for total T3 or total T4. The authors also confirmed that Hgb levels normalized in almost all patients with GD anaemia usually $16 \pm 6.3$ weeks of ATD therapy [33]

In our study, the number of patients with anaemia at diagnosis of GD was comparable to the above-mentioned findings and we also noticed a significant increase in $\mathrm{Hgb}$ levels after the initiation of ATD therapy, even though in our study the observation period was shorter. Due to the small number of boys in our study group we did not analyse our results in gender-related subgroups. In the earlier study by Reddy et al. [34], the most common abnormality of the peripheral blood picture found in patients with uncomplicated thyrotoxicosis was the erythrocytes microcytosis, found in $37 \%$ of patients. Anaemia was reported in $8.5 \%$, absolute lymphocytosis in $11 \%$ and neutropenia in $2.5 \%$ of patients. In our study group, decreased MCV value was also found in more than one-third of children, and it did not change significantly after the first few weeks of ATD therapy. Geetha and Srikrishna [35] evaluated RDW and MCV in patients with thyroid dysfunction and found that RDW values were significantly increased in both hypo- and hyperthyroid patients, while MCV was significantly decreased in hyperthyroidism and significantly increased in hypothyroidism. The authors concluded that abnormal levels of thyroid hormones might substantially influence the size variability of circulating RBC [35]. Our analysis also confirmed significant changes in RDW values, which were increased in $15.3 \%$ of untreated GD children and in 51.3\% after the first 4-6 weeks of ATD therapy. Kawa et al. [19], who aimed to explain the exact mechanism of thyroid hormones' action on human haematopoiesis, showed that both hypo- and hyperthyroidism modify thyroid hormone receptors gene expression in haematopoietic progenitor cells (HPCs) in vivo. Their investigations detected an increase in the frequency of apoptotic CD34(+)-enriched HPCs in both hypo- and hyperthyroidism with a modulation of apoptosis-related genes. They concluded that the molecular mechanism by which thyroid hormones influence haematopoiesis might provide a basis for designing novel therapeutic interventions in thyroid diseases [19]. The complexity of those mechanisms may explain why we did not find any direct associations between free TH levels and red blood cell parameters in our patients. Changes in WBC and PLT pictures in patients with GD are less frequent than RBC abnormalities. The incidence of neutropenia in thyrotoxicosis is estimated at $2.5-18 \%[34,36,37]$. Our analysis showed that WBC and neutrophil counts, more often than PLT count, were decreased in patients with GD, both untreated and during the first weeks of ATD therapy. None of the patients had severe leukopenia or thrombocytopenia. We also found that after the initiation of ATD therapy, PLT count normalized faster than WBC count, which remained unchanged after the first 4-6 weeks of treatment. We also noted that more than one-third of children had (usually slightly) decreased 
neutrophil count before the initiation of ATD therapy, which normalized in most of them during ATD treatment, and remained slightly decreased only in $10.7 \%$ of patients. Our findings do not coincide with earlier results by Dorgalaleh et al. [11], who confirmed significant changes in red blood cell parameters, such as Hgb and RDW, but did not find any statistically significant differences in WBC or PLT counts between hyperthyroid and hypothyroid patients. Authors which confirmed decreased PLT count in patients with GD, suggest that thrombocytopenia in that group could be related to an increased sequestration potency of the reticuloendothelial phagocyte system stimulated by thyroid hormone [38]. It is worthy to note that although the initiation of ATD therapy usually results in normalization of the Hgb level and RBC, WBC and PLT counts, neutropenia and agranulocytosis may occur as adverse effects of ATD therapy, which indicates the need of constant monitoring of CBC in GD patients [13, 14, 33].

The second part of our study was the analysis of the relationships between thyroid hormone concentrations, autoimmune process activity and aminotransferase activity both at baseline, before the initiation of therapy, and after 4-6 weeks of ATD therapy. The coincidence of liver dysfunction and hyperthyroidism is relatively frequent, but varies significantly between studies [27, 39]. Hepatic dysfunctions in patients with hyperthyroidism are related to the effects of thyroid hormone excess, ATD-related liver injury and to the presence of concomitant liver disease [40, 41]. Our study showed increased ALT activity in 44\% of children and increased AST activity in $32.2 \%$ of patients before the initiation of ATD therapy. Elevations of ALT and AST activity were more than twice above the upper normal range in $6.8 \%$ and in $5 \%$ of patients for ALT and AST, respectively. In the literature, as in the case of haematologic disorders, data concerning the incidence of liver dysfunctions among paediatric patients with GD are limited. Biscoveanu and Hasinski [42], who aimed to determine the incidence of liver dysfunction in patients with hyperthyroidism, noticed abnormal results of a liver function test in $37 \%$ of adult patients. They found increased ALT activity in $26 \%$ of patients and increased AST activity in $17 \%$. Some studies indicate that hyperthyroidism leads mainly to an increase in ALT activity, while AST activity is not affected by thyroid hormone excess to such degree [43]. Our findings in paediatric populations with GD corresponded with the above-mentioned results. On the other hand, Targher et al. [44], who investigated the relationships between serum ALT activity and thyroid function tests in a cohort of hypothyroid, euthyroid and hyperthyroid adult individuals, reported that ALT activity increased significantly steadily across the increasing TSH concentrations and simultaneously there was a negative relationship between serum ALT activity and serum fT4 levels [44]. Despite some discrepancies in the literature, an increase in serum liver enzyme activity seemed to be relatively common. Liver injury related to untreated hyperthyroidism usually normalizes during therapy [39]. In our study, 4-6 weeks after the initiation of ATD therapy we noticed a significant decrease in ALT activity, while AST activity did not change significantly. We also found that ALT activity was related to baseline TSH and free TH levels. Simultaneously, we did not find any significant associations between autoimmune process activity and aminotransferase activity, which could suggest that it is a thyroid hormone excess, rather than the autoimmune process itself, that is responsible for hepatic enzymes elevation.

The results of our study indicate that baseline screening for haematologic abnormalities and liver dysfunction should be a standard practice in all newly diagnosed children and adolescents with GD before the initiation of ATD therapy. Although changes in haematopoiesis and liver function are usually mild and reversible during ATD therapy, due to possible side effects of such treatment, routine monitoring of peripheral blood picture and aminotransferase activity should also be performed during ATD therapy.

\section{Conclusions}

Changes in haematopoiesis and liver function are relatively frequent in children and adolescents with newly diagnosed GD. Their incidence seems to be similar to that reported in adult population with GD. The most common alterations seem to be those in neutrophil count, red blood cell parameters and in ALT activity. The initiation of ATD therapy usually leads to a significant improvement in those parameters as early as in the first few weeks of treatment.

\section{The authors declare no conflict of interest.}

\section{References}

1. Lavard L, Ranlřv I, Perrild H, et al. (1994): Incidence of juvenile thyrotoxicosis in Denmark, 1982-1988. A nationwide study. Eur J Endocrinol 130: 565-568.

2. Kahaly GJ, Bartalena L, Hegedüs L, et al. (2018): 2018 European Thyroid Association Guideline for the Management of Graves' Hyperthyroidism. Eur Thyroid J 7: 167-186.

3. Hadj-Kacem H1, Rebuffat S, Mnif-Féki M, et al. (2009): Autoimmune thyroid diseases: genetic susceptibility of thyroid-specific genes and thyroid autoantigens contributions. Int J Immunogenet 36: 85-96.

4. Brix TH, Kyvik KO, Hegedüs L (1998): What is the evidence of genetic factors in the etiology of Graves' disease? A brief review. Thyroid 8: 727-734.

5. Brand OJ, Gough SC (2010): Genetics of thyroid autoimmunity and the role of the TSHR. Mol Cell Endocrinol 322: 135-143.

6. Brix TH, Hansen PS, Kyvik KO, Hegedus L (2000): Cigarette smoking and risk of clinically overt thyroid disease: a population-based twin case-control study. Arch Intern Med 160: 661-666. 
7. DeGroot LJ. Diagnosis and Treatment of Graves' Disease (Updated 2016 Nov 2): In: Endotext [Internet], De Groot LJ, Chrousos G, Dungan K, et al. (eds.). MDText.com Inc., South Dartmouth (MA). Available from: https://www.ncbi.nlm.nih. gov/books/NBK285548/

8. Fröhlich E, Wahl R (2017): Thyroid autoimmunity: role of anti-thyroid antibodies in thyroid and extra-thyroidal diseases. Front Immunol 8: 521.

9. Weetman AP (2000): Graves' disease. N Engl J Med 343: 1236-1248

10. Malik R, Hodgson H (2002): The relationship between the thyroid gland and the liver. QJM 95: 559-569.

11. Dorgalaleh A, Mahmoodi M, Varmaghani B, et al. (2013): Effect of thyroid dysfunction on blood cell count and red blood cell indice. Iran J Ped Hematol Oncol 3: 73-77.

12. Elias RM, Dean DS, Barsness GW (2012): Hepatic dysfunction in hospitalized patients with acute thyrotoxicosis: a decade of experience. ISRN Endocrinol 2012: 325092.

13. Cooper DS (2003): Antithyroid drugs in the management of patients with Graves' disease: an evidence-based approach to therapeutic controversies. J Clin Endocrinol Metab 88: $3474-$ 3481.

14. Cooper DS (2005): Antithyroid drugs. N Engl J Med 352: 905-917.

15. Rivkees SA, Mattison DR (2009): Ending propylthiouracil-induced liver failure in children. N Engl J Med 360: 1574-1575.

16. Rivkees SA, Szarfman A (2010): Dissimilar hepatotoxicity profiles of propylthiouracil and methimazole in children. J Clin Endocrinol Metab 95: 3260-3267.

17. Nakamura H, Noh JY, Itoh K, et al. (2007): Comparison of methimazole and propylthiouracil in patients with hyperthyroidism caused by Graves' disease. J Clin Endocrinol Metab 92: 2157-2162.

18. Shaw B, Mehta AB (2002): Pancytopenia responding to treatment of hyperthyroidism: a clinical case and review of literature. Clin Lab Haematol 24: 385-387.

19. Kawa MP, Grymuła K, Paczkowska E, et al. (2010): Clinical relevance of thyroid dysfunction in human haematopoiesis: biochemical and molecular studies. Eur J Endocrinol 162: 295-305.

20. Golde DW, Bersch N, Chopra IJ (1977): Thyroid hormones stimulate erythropoiesis in vitro. Br J Haematol 37: 173-177.

21. Naji P, Kumar G, Dewani S, et al. (2013): Graves' disease causing pancytopenia and autoimmune hemolytic anemia at different time intervals: a case report and a review of the literature. Case Rep Med 2013: 194542.

22. Pincet L, Gorostidi F (2018): Graves' disease causing pancytopenia: case report and literature review. Clin Med Insights Case Rep 11: 1179547618781090.

23. Ford HC, Carter JM (1988): The haematology of hyperthyroidism: abnormalities of erythrocytes, leucocytes, thrombocytes and haemostasis. Postgrad Med J 64: 735-742.

24. Tomczyńska M, Saluk-Bijak J (2018): The mutual cooperation of blood platelets and lymphocytes in the development of autoimmune thyroid diseases. Acta Biochim Pol 65: 17-24.

25. Choudhary AM, Roberts I (1999): Thyroid storm presenting with liver failure. J Clin Gastroenterol 29: 318-321.

26. Mansourian AR (2013): Liver functional behaviour during thyrotoxicosis: a review. J Biol Sci 13: 665-678.

27. Lin TY, Shekar AO, Li N, et al. (2017): Incidence of abnormal liver biochemical tests in hyperthyroidism. Clin Endocrinol (Oxf) 86: 755-759.
28. Bayraktar M, Van Thiel DH (1997): Abnormalities in measures of liver function and injury in thyroid disorders. Hepatogastroenterology 44: 1614-1618.

29. Thompson P, Strum D, Boehm T, et al. (1978): Abnormalities of liver function tests in thyrotoxicosis. Military Medicine 143: 548-551.

30. Levy M (1993): Propylthiouracil hepatotoxicity: a review and case presentation. Clin Pediatr (Phila) 32: 25-29.

31. Hambsch K, Herrmann F, Fischer H, et al. (1989): [Changes in the blood picture in hyperthyroidism]. Z Gesamte Inn Med 44: 300-306.

32. Orwoll ES, Orwoll RL (1987): Hematologic abnormalities in patients with endocrine and metabolic disorders. Hematol Oncol Clin North Am 1: 261-279.

33. Gianoukakis AG, Leigh MJ, Richards P, et al. (2009): Characterization of anemia associated with Graves' disease. Clin Endocrinol (Oxf) 70: 781-787.

34. Reddy J, Brownlie BE, Heaton DC, et al. (1981): The peripheral blood picture in thyrotoxicosis. N Z Med J 93: 143-145.

35. Geetha JP, Srikrishna R (2012): Role of red blood cell distribution width (rdw) in thyroid dysfunction. Int J Biol Med Res. 3: 1476-1478.

36. Irvine WJ, Wu FC, Urbaniak SJ, Toolis F (1977): Peripheral blood leucocytes in thyrotoxicosis (Graves' disease) as studied by conventional light microscopy. Clin Exp Immunol 27: 216-221.

37. Eakin DL, Peake RL, Weiss GB (1983): Effect of therapy on the neutropenia of hyperthyroidism. South Med J 76: 335$337,340$.

38. Kurata Y, Nishioeda Y, Tsubakio T, Kitani T (1980): Thrombocytopenia in Graves' disease: effect of T3 on platelet kinetics. Acta Haematol 63: 185-190.

39. Fong TL, McHutchinson JG, Reynolds TB (1992): Hyperthyroidism and hepatic dysfunction. A case series analysis. J Clin Gastroenterol 14: 240-244.

40. Williams KV, Nayak S, Becker D, et al. (1997): Fifty years of experience with propylthiouracil-associated hepatotoxicity: what have we learned? J Clin Endocrinol Metab 82: 17271733 .

41. Wang R, Tan J, Zhang G, et al. (2017) Risk factors of hepatic dysfunction in patients with Graves' hyperthyroidism and the efficacy of 131iodine treatment. Medicine 96: 5 .

42. Biscoveanu M, Hasinski S (2000): Abnormal results of liver function tests in patients with Graves' disease. Endocr Pract 6: 367-369.

43. Madani SH, Far ZR, Jalilian N, et al. (2014): Evaluate the liver function in hyperthyroidism patients. J Paramed Sci 5: 75-78.

44. Targher G, Montagnana M, Salvagno G, et al. (2008): Association between serum TSH, free T4 and serum liver enzyme activities in large cohort of unselected outpatients. Clin Endocrinol (Oxf) 68: 481-484. 